\title{
Eficacia de los objetos virtuales para el aprendizaje en el uso de estrategias de lectura de estudiantes de distritos del Perú con restricciones en conectividad y equipamiento - caso Villa Rica
}

\author{
Efficacy of learning virtual objects in the use of reading strategies \\ of students in districts of peru with restrictions on connectivity and \\ computer equipment - the Villa Rica case
}

Jorge ERnesto TorRes-ObleAs*

Daniela Medina-Coronado**

\section{Resumen}

El uso de tecnologías de información y comunicación en el Perú ha contribuido a mayor acceso a la educación y al desarrollo humano. Sin embargo, existen poblaciones que, al carecer de conectividad, no gozan completamente esas oportunidades. Mediante una propuesta cuasi experimental, se busca determinar el efecto del nivel de interactividad de los objetos virtuales para el aprendizaje, OVA, en la adquisición de estrategias de lectura; relevante para el diseño de OVA, al estar la interactividad condicionada por la conectividad. Se intervino un grupo experimental con OVA de interactividad pasiva y otro, con OVA de interactividad limitada, así como un grupo control; conformados por estudiantes del distrito de Villa Rica que accedieron a la plataforma virtual Clementina. Ambos OVA posibilitaron mejoras significativas al usar estrategias de lectura; aunque se evidenciaron mayores puntuaciones en el grupo con OVA de interactividad limitada, no existieron diferencias significativas en la eficacia de los OVA.

Palabras clave: diseño de objetos virtuales para el aprendizaje, estrategias de lectura.

\footnotetext{
*Universidad César Vallejo, Lima, Perú. E-mail: jtorreso@ucv.edu.pe (D) orcid.org/0000-0002-8537-1622 Google Scholar

*** Universidad César Vallejo, Lima, Perú. E-mail: dmedinac@ucv.edu.pe

(D) orcid.org/0000-0002-9180-7613 Google Scholar
} 


\begin{abstract}
The use of information and communication technologies in Peru has contributed to greater access to education and human development. However, there are populations that, lacking connectivity, do not fully enjoy these opportunities. Through a quasi-experimental proposal, the objective of this research is to determine the effect of the level of interactivity of learning virtual objects, LVOs, in the acquisition of reading strategies which is relevant for the design of the LVOs since interactivity is conditioned by connectivity. An experimental group was intervened with LVOs of passive interactivity and another, with LVOs of limited interactivity, as well as a control group conformed by students of the district of Villa Rica who accessed the virtual platform Clementina. Both LVOs enabled significant improvements in the use of reading strategies. Although higher scores were evident in the limited interactivity LVO group, there were no significant differences in the efficacy of LVOs.
\end{abstract}

Key words: design of learning virtual objects for learning, reading strategies.

\title{
Introducción
}

La educación viene presentando cambios constantes y drásticos desde inicios del presente siglo, con respecto a las formas de enseñar y de aprender, originados principalmente por el uso de las tecnologías de la información y comunicación TIC, de forma globalizada. Las escuelas deben preparar a los estudiantes para la vida e inclusive para empleos que aún no existen y, para afrontar estos retos, las competencias digitales adquieren vital importancia, así como también la necesidad de insertar medios y recursos digitales a los procesos de enseñanza aprendizaje.

En el caso peruano, de acuerdo con el IMD World Competitiveness Center (2018) en su ranking de Competitividad digital mundial 2018, Perú se sitúa entre los últimos lugares de los países participantes; entre los países de Latinoamérica, por debajo de Chile (puesto 37) y Colombia (puesto 59), y solo por encima de Venezuela (puesto 63). Dicha medición busca evaluar la capacidad de un país para adoptar tecnologías digitales en cuanto a conocimientos, tecnologías y preparación para el futuro. Los resultados muestran que el Perú ha retrocedido en estos indicadores, principalmente en cuanto a preparación para el futuro, ubicándose en el puesto 60 de 63 economías estudiadas.

Asimismo, considerando el índice de conocimiento (IK) propuesto por el Banco Mundial para el 2018, y basado en los índices sectoriales: formación preuniversitaria; formación técnico profesional, formación superior, tecnología de la información y comunicación, investigación, desarrollo e innovación y economía; Perú se encuentra por debajo de la media (48) con un 
Eficacia de los objetos virtuales para el aprendizaje en el uso de estrategias de lectura de estudiantes de distritos del Perú...

IK de 43 y en el puesto 86 de 134 países; identificándose como puntos críticos: la formación técnica profesional, las tecnologías de la información y la comunicación, la economía y la educación superior. Uno de los aspectos aún por mejorar en la educación superior, según el informe bienal sobre la realidad universitaria peruana del Ministerio de Educación peruano (Minedu, 2017) -aunque el informe del Banco Mundial no lo considera- es el acceso a educación superior universitaria: en el año 2014 se situó en un 41\%, con un considerable 59\% de jóvenes que no tienen esa posibilidad. Y si se considera al quintil más pobre, el acceso es solo del $17 \%$.

Una alternativa para solucionar estas brechas de acceso a la educación universitaria - por causas físicas o económicas - es la educación virtual, posibilitando la ampliación de la cobertura educativa (Yong, Nagles, Mejía y Chaparro, 2017).

La educación virtual trabaja con recursos digitales, entendidos como cualquier elemento en formato digital destinado a apoyar el proceso de aprendizaje sobre algún tema, como aplicaciones, programas, textos, multimedia, videos, audios, entre otros. Estos recursos digitales no cuentan necesariamente con secuencias didácticas, diseño para la instrucción o secuencias formativas específicas; lo que justifica la necesidad de objetos virtuales de aprendizaje (OVA) que sí poseen una secuencia formativa y que pueden, si fuera necesario, llegar a ser elementos dentro de una estructura mayor.

En ese marco, se plantea el proyecto "Acceso a carreras profesionales en distritos sin servicios universitarios" de la Universidad César Vallejo que, a través de la educación a distancia en entornos virtuales, busca acercar la educación superior universitaria en Perú a lugares en donde no ha tenido presencia; esto, a partir de un estudio preliminar en algunos distritos sobre las condiciones tecnológicas, acceso, uso y manejo de TIC en los jóvenes, entre otras variables, con el propósito de diseñar y elaborar recursos de aprendizaje con el respectivo soporte tecnológico.

Al considerar estas variables, durante el correspondiente proceso de diseño instruccional surgió una interrogante, conocer si la variable nivel de interactividad de los objetos virtuales para el aprendizaje (OVA) que se estaban elaborando tenía algún efecto en el aprendizaje, a fin de probar y optimizar los OVA desarrollados para atender a jóvenes ingresantes a la universidad, pertenecientes a distritos donde no existe presencia física de la universidad y cuyo nivel de conectividad a Internet, por lo general, presenta dificultades. Diversos estudios refieren que la interactividad es un aspecto relevante en la motivación para aprender y que los estudiantes sienten que es más beneficiosa, tal como refieren estudios como los de White y Sykes (2012); Snowball (2014); Feria y Zúñiga (2016); más oportunidades de aprendizaje interactivo, como el marcado personal en línea, la selección múltiple, videos, juegos y los ejercicios gráficos tienen un mayor impacto en el rendimiento de los estudiantes que actividades pasivas, como diapositivas, audios, imágenes con texto o textos planos, concluyéndose que los 
recursos más planos y menos interactivos son los de menor acogida. Un estudio vinculado a dicha problemática que involucró a más de 17.000 estudiantes de tres universidades españolas realizado por Castaño, Duart y Vinuesa (2014), concluyen que aumentar el tiempo dedicado a estudiar en línea es útil cuando se trata de alguna forma de interactividad.

Se encontraron pocos estudios que pretendían comparar los efectos en el aprendizaje según nivel de interactividad, uno de ellos el de Guthrie, Vallée-Tourangeau, Vallée-Tourangeau. y Howard (2015), halló efectos positivos en la resolución de problemas, en experimentos donde usaban tanto alta como baja interactividad.

Se pensó en qué tipo de habilidades deben poseer los jóvenes que egresan de la educación básica regular para tener un aprendizaje efectivo y teniendo como un aspecto relevante la comprensión lectora, se propuso realizar un estudio experimental para probar la eficacia de los OVA desarrollados en la adquisición de estrategias de comprensión lectora en función del contexto de su aplicación - especialmente, nivel de conectividad y equipamiento disponiblesa fin de proponer sugerencias para su elaboración.

Todo lector necesita tener habilidades que le permitan adaptarse estratégicamente a las características de determinado texto y a los requerimientos de la lectura y seguir un conjunto de pasos que le permitan enfrentarse de forma efectiva a un texto. Al respecto, Gutiérrez y Salmerón (2012) indican que las estrategias de lectura son similares a una toma de decisión, seleccionando procedimientos de aprendizaje para facilitar una lectura fluida, intencional, activa y comprometida con la meta y las características específicas del texto. Por su lado, Solé (2011), una de las investigadoras principales en este tema, afirma que las estrategias de lectura implican el cumplimiento de objetivos y la planificación de acciones para lograr los objetivos del lector y evaluar un posible cambio.

En el presente estudio, las estrategias de lectura abordadas y evaluadas fueron las propuestas por Solé (2011): estrategias antes de la lectura, que buscan crear la necesidad de leer y brindarle al lector los recursos adecuados para empezar la lectura con mucha seguridad; aquellas que vuelven al lector, un lector activo. Una de las estrategias más usadas es la prelectura, que consiste en explorar rápidamente el texto para conocer su estructura y tener una idea general del mismo, permitiendo decidir la importancia de la lectura. Asimismo, las estrategias durante la lectura, a través de actividades que permiten a los estudiantes desarrollar habilidades para realizar inferencias de acuerdo con los diferentes textos, hacer preguntas sobre el tema en cuestión y evaluar y comprobar su propia comprensión; es decir, para monitorear el proceso. Finalmente, las estrategias después de la lectura, mediante la revisión de palabras subrayadas, notas agregadas al texto y apuntes realizados durante la lectura, para comprobar que tengan sentido y organizarlos en resúmenes, diagramas, fichas y esquemas, lo que permitirá la síntesis y comprensión total del texto leído (Sánchez, 2009). 
Eficacia de los objetos virtuales para el aprendizaje en el uso de estrategias de lectura de estudiantes de distritos del Perú...

\section{Objetivo y método}

El objetivo planteado en el estudio fue conocer el efecto del uso de objetos virtuales para el aprendizaje en las estrategias de lectura utilizadas por los estudiantes del quinto año de secundaria de IE públicas del distrito de Villa Rica, provincia y departamento de Pasco, en la perspectiva de determinar si existen diferencias en el nivel de adquisición de estas estrategias, según el nivel de interactividad de los OVA aplicados.

La investigación se concibió bajo un enfoque cuantitativo, con un diseño cuasi experimental. La muestra estuvo conformada por 123 estudiantes pertenecientes al quinto de secundaria de tres instituciones educativas públicas del distrito de Villa Rica en la Provincia de Oxapampa, Región Pasco: 59 del primer grupo experimental, cuya intervención se realizó con objetos virtuales de interactividad pasiva o sin interactividad, OVA "A"; 41 estudiantes del segundo grupo experimental con objetos virtuales de interactividad limitada, OVA “B” y 23 estudiantes que formaron parte del grupo control.

Las instituciones educativas públicas con jornada escolar completa fueron Leopoldo Krause, Pedro Ruiz Gallo y Santa Polonia; pertenecientes a Villa Rica, un distrito rural que no cuenta con servicios de educación superior y cuya población tiene restricciones en conectividad y equipamiento para el acceso a Internet. Además de estas condiciones relevantes para el experimento, también se consideró que en los resultados de la Evaluación Censal de Estudiantes del año 2016, solo el 9,8\% los estudiantes del distrito se ubicaron en nivel satisfactorio de comprensión lectora y un alarmante $91,2 \%$, entre los niveles previo inicio, inicio y proceso. Se trata de resultados que evidencian una necesidad de intervención (Minedu, 2017).

Para el experimento, se habilitaron aulas virtuales en una plataforma LMS, asignadas para cada tipo de intervención; ambas aulas experimentales contaron con el acompañamiento de un tutor virtual que tuvo la función de realizar la correspondiente retroalimentación a las actividades propuestas y de absolver dudas o reforzar aspectos que no quedaran claros durante el trabajo autónomo, así como con los coordinadores del área de comunicación y del aula de innovación pedagógica, quienes tenían la función de realizar el recordatorio respectivo a los estudiantes para el ingreso al aula virtual y de habilitar la sala del cómputo en la IE, para que los estudiantes puedan realizar las actividades de aprendizaje según lo requieran. Esto último en consideración de que en la zona de estudio existen problemas de conectividad y de acceso a la tecnología, por aspectos de carácter socioeconómico y de ubicación geográfica.

Las dos aulas virtuales habilitadas tuvieron los mismos recursos virtuales, pero se diferenciaron en la forma de presentar de los objetos para el aprendizaje. En efecto, en una de ellas se encontraban videos, lecturas, contenidos, encapsulados, granulados en un solo recurso SCORM con cierta interactividad; en la otra se encontraban los mismos objetos, pero sin 
encapsular, sueltos y sin interactividad. En ambos casos, además de los objetos mencionados, contaron con autoevaluaciones y actividades semanales. La intervención tuvo una duración de cinco semanas: cuatro semanas para la revisión de sendos temas propuestos y una adicional para la culminación de las actividades. Los cuatro temas estuvieron basados en las estrategias para la lectura de Solé (2011): estrategias antes, durante y después de la lectura y ejercicios de aplicación completa a partir de textos expositivos.

Se determinaron las condiciones iniciales de los grupos experimentales y del grupo control con una prueba de homogenización, hallándose que no existían diferencias entre los grupos de estudio. El instrumento para la recolección de datos en el pre y post test fue el examen escrito, elaborado en función de la propuesta del Ministerio de Educación para quinto de secundaria, con el propósito de evaluar el uso de estrategias de comprensión lectora. La validez de contenido del instrumento estuvo a cargo de especialistas del área, quienes emitieron el juicio de "aplicable" con una V de Aiken de 0,96 y un nivel de confiabilidad de 0,657 Alfa de Cronbach; en el caso de una prueba de rendimiento académico como en el caso del presente instrumento, la literatura considera como aceptable un coeficiente desde 0,61 (Thorndike y Magnusson como se citaron en Ruiz, 1992). Luego de recolectados los datos y determinado el comportamiento de estos con la prueba Shapiro-Wilk, los resultados hallados determinaron que la prueba de hipótesis se realice con la prueba no paramétrica U de Mann-Whitney.

\section{Resultados}

Tabla 1. Resultados descriptivos del pre y postest del uso de estrategias de lectura y sus dimensionesOVA A grupo experimental.

\begin{tabular}{|c|c|c|c|c|c|c|}
\hline & $\mathrm{N}$ & Rango & Mínimo & Máximo & Media & $\begin{array}{l}\text { Desviación } \\
\text { estándar }\end{array}$ \\
\hline Estrategias de lectura pretest OVA A & 41 & 5 & 1 & 6 & 3,71 & 1,230 \\
\hline Estrategias de lectura post-test OVA A & 41 & 11 & 3 & 14 & 8,68 & 2,444 \\
\hline Estrategias antes de la lectura pretest OVA A & 41 & 5 & 0 & 5 & 1,80 & 1,100 \\
\hline Estrategias antes de la lectura post-test OVA A & 41 & 5 & 1 & 6 & 3,80 & 1,418 \\
\hline Estrategias durante de la lectura pretest OVA A & 41 & 3 & 0 & 3 & ,44 & ,709 \\
\hline Estrategias durante de la lectura post-test OVA A & 41 & 6 & 0 & 6 & 1,71 & 1,401 \\
\hline Estrategias después de la lectura pretest OVA A & 41 & 3 & 0 & 3 & 1,46 &, 596 \\
\hline Estrategias después de la lectura post-test OVA A & 41 & 6 & 0 & 6 & 3,17 & 1,465 \\
\hline $\mathrm{N}$ válido (por lista) & 41 & & & & & \\
\hline
\end{tabular}

Fuente: elaboración propia.

En la tabla 1 se muestran los resultados descriptivos del grupo experimental que trabajó con el OVA “A”, de interactividad baja. Los resultados obtenidos en el pre test muestran un puntaje 
Eficacia de los objetos virtuales para el aprendizaje en el uso de estrategias de lectura de estudiantes de distritos del Perú...

medio de 3,71 de un total de 18 puntos, a diferencia de los valores obtenidos en el post test, donde se evidencia un puntaje promedio de 8,68 puntos, incrementándose en 4,97 puntos; es decir, en un 134\%, con respecto al uso de estrategias de lectura. A pesar de que en el resultado pos-test se evidencian mejoras en el uso de estrategias de lectura, en comparación con el puntaje total de la prueba, lo obtenido no alcanza el 50\% del puntaje total.

Al realizar el análisis descriptivo de las tres dimensiones de la variable dependiente, se observa que en la dimensión 1 "Estrategias antes de la lectura" de 1,80 puntos obtenidos en el pretest, en el postest mejoró el puntaje a 3,80 puntos de un total de 6 puntos, incrementándose las puntuaciones en un $111 \%$. Con relación a la dimensión 2 "Estrategias durante la lectura", de 0,44 se incrementó a 1,71 de un total de 6 puntos; evidenciándose un incremento de $289 \%$ en el valor de la media. Y finalmente, en la dimensión 3 "Estrategias después de la lectura", de 1,46 puntos en el pretest, se mejoró a 3,17 puntos en el postest de un total de 6 puntos, observándose una mejora del $117 \%$ en el valor de la media aritmética.

Tabla 2. Resultados descriptivos del pre y postest del uso de estrategias de lectura y sus dimensiones OVA B - grupo experimental.

\begin{tabular}{|c|c|c|c|c|c|c|}
\hline & $\mathbf{N}$ & Rango & Mínimo & Máximo & Media & $\begin{array}{l}\text { Desviación } \\
\text { estándar }\end{array}$ \\
\hline Estrategias de lectura pretest OVA B & 59 & 5 & 1 & 6 & 3,69 & 1,004 \\
\hline Estrategias de lectura post- test OVA B & 59 & 15 & 3 & 18 & 9,41 & 2,743 \\
\hline Estrategias antes de la lectura pretest OVA B & 59 & 3 & 0 & 3 & 1,73 & ,665 \\
\hline Estrategias antes de la lectura postest OVA B & 59 & 4 & 2 & 6 & 4,31 & 1,263 \\
\hline Estrategias durante la lectura pretest OVA B & 59 & 1 & 0 & 1 & ,41 & ,495 \\
\hline Estrategias durante la lectura postest OVA B & 59 & 6 & 0 & 6 & 1,86 & 1,570 \\
\hline $\begin{array}{l}\text { Estrategias después de la lectura pretest } \\
\text { OVA B }\end{array}$ & 59 & 3 & 0 & 3 & 1,56 & ,623 \\
\hline $\begin{array}{l}\text { Estrategias después de la lectura postest } \\
\text { OVA B }\end{array}$ & 59 & 5 & 1 & 6 & 3,24 & 1,236 \\
\hline N válido (por lista) & 59 & & & & & \\
\hline
\end{tabular}

Fuente: elaboración propia. 
De acuerdo con lo mostrado en la tabla 2, se observa que el promedio obtenido de la variable dependiente "uso de estrategias de lectura" en el pretest es 3,83 de un total de 18 puntos, mientras que en el postest se obtuvo una media de 9,41, evidenciándose un incremento de 5,58 puntos, que equivale a un 145\% por la aplicación del OVA "B" de interactividad limitada. Asimismo, los resultados descriptivos de las 3 dimensiones de la variable dependiente evidencian que luego de la aplicación, se han incrementado las puntuaciones en el postest. En efecto, en el caso de la dimensión 1 "estrategias antes de la lectura" el promedio de los datos obtenidos pasó de 1,73 a 4,31 puntos de un total de 6 puntos, representando un incremento del $149 \%$. En cuanto a la segunda dimensión "estrategias durante la lectura", los resultados obtenidos reflejan que la aplicación del OVA "B" ha mejorado los resultados iniciales en un $29,5 \%$, logrando que el promedio del pretest de 0,44 puntos se incremente a 0,57 puntos de un total de 6 puntos. Asimismo, en la dimensión 3 "estrategias después de la lectura” también se constata un incremento en el promedio obtenido, de 1,56 a 3,24 puntos que representa un incremento de $107,7 \%$.

Tabla 3. Prueba de hipótesis para el postest OVA tipo A.

\begin{tabular}{lrrrr}
\hline & $\begin{array}{c}\text { Uso de estrategias } \\
\text { de lectura OVA A }\end{array}$ & $\begin{array}{c}\text { Estrategias antes de la } \\
\text { lectura OVA A }\end{array}$ & $\begin{array}{c}\text { Estrategias durante la } \\
\text { lectura OVA A }\end{array}$ & $\begin{array}{c}\text { Estrategias después } \\
\text { de la lectura } \\
\text { OVA A }\end{array}$ \\
\hline U de Mann-Whitney & 112,500 & 278,500 & 225,500 & 176,500 \\
W de Wilcoxon & 412,500 & 578,500 & 525,500 & 476,500 \\
Z & $-5,121$ & $-2,856$ & $-3,684$ & $-4,299$ \\
Sig. asintót. (bilateral) &, 000 &, 004 &, 000 &, 000 \\
& & a. Variable de agrupación: Grupos. &
\end{tabular}

Fuente: elaboración propia.

Los resultados obtenidos, luego de la aplicación de los programas de intervención que se muestran en las tablas 3 y 4, tanto para el uso del OVA “A” de interactividad pasiva como para el OVA "B" de interactividad limitada, muestran valores de significación asintótica menores al nivel alfa de 0,05 , demostrando que ambos programas han producido mejoras significativas en el uso de estrategias de lectura y en cada una de sus dimensiones en las muestras intervenidas con respecto al grupo control. 
Eficacia de los objetos virtuales para el aprendizaje en el uso de estrategias de lectura de estudiantes de distritos del Perú...

Tabla 4. Prueba de hipótesis para el postest OVA tipo B.

\begin{tabular}{|c|c|c|c|c|}
\hline & $\begin{array}{c}\text { Uso de } \\
\text { Estrategias de } \\
\text { lectura OVA B }\end{array}$ & $\begin{array}{c}\text { Estrategias antes } \\
\text { de la lectura } \\
\text { OVA B }\end{array}$ & $\begin{array}{c}\text { Estrategias } \\
\text { durante la } \\
\text { lectura OVA B }\end{array}$ & $\begin{array}{c}\text { Estrategias } \\
\text { después de la } \\
\text { lectura OVA B }\end{array}$ \\
\hline $\begin{array}{l}\text { U de Mann- } \\
\text { Whitney }\end{array}$ & 75,000 & 256,500 & 337,500 & 149,000 \\
\hline $\begin{array}{l}\text { W de } \\
\text { Wilcoxon }\end{array}$ & 351,000 & 532,500 & 613,500 & 425,000 \\
\hline $\mathrm{Z}$ & $-6,264$ & $-4,439$ & $-3,641$ & $-5,601$ \\
\hline $\begin{array}{l}\text { Sig. asintót. } \\
\text { (bilateral) }\end{array}$ &, 000 & ,000 & ,000 & ,000 \\
\hline
\end{tabular}

a. Variable de agrupación: Grupos

Fuente: elaboración propia.

Tabla 5. Prueba de hipótesis sobre la eficacia en el uso de estrategias de lectura entre el OVA A y el OVA B.

\begin{tabular}{|c|c|c|c|c|}
\hline & $\begin{array}{c}\text { Uso de } \\
\text { estrategias de } \\
\text { lectura }\end{array}$ & $\begin{array}{c}\text { Estrategias antes } \\
\text { de la lectura }\end{array}$ & $\begin{array}{c}\text { Estrategias } \\
\text { durante la lectura }\end{array}$ & $\begin{array}{c}\text { Estrategias después } \\
\text { de la lectura }\end{array}$ \\
\hline $\begin{array}{l}\text { U de } \\
\text { Mann- } \\
\text { Whitney }\end{array}$ & 1054,000 & 975,500 & 1163,500 & 1193,500 \\
\hline $\begin{array}{l}\text { W de } \\
\text { Wilcoxon }\end{array}$ & 1915,000 & 1836,500 & 2024,500 & 2054,500 \\
\hline $\mathrm{Z}$ & $-1,099$ & $-1,680$ &,- 332 &,- 115 \\
\hline $\begin{array}{l}\text { Sig. } \\
\text { asintót. } \\
\text { (bilateral) }\end{array}$ &, 272 & ,093 & ,740 & ,908 \\
\hline
\end{tabular}

a. Variable de agrupación: Grupos.

Fuente: elaboración propia. 
Luego de aplicar los objetos virtuales para el aprendizaje tipo A y B en las respectivas muestras y evaluar la variable dependiente uso de estrategias de lectura, se observa que la significación asintótica para la variable y cada una de sus dimensiones son mayores a 0,05; indicando que no existen diferencias significativas en los resultados obtenidos entre los dos grupos experimentales. Es decir, el nivel de interactividad de los OVA utilizados en el estudio, no han significado mayores diferencias en el uso de estrategias de lectura. No obstante, en el análisis descriptivo, se pudo observar que las puntuaciones promedio del uso de estrategias de lectura del OVA B son mejores que los del OVA A.

\section{Discusión}

Algunas limitaciones que probablemente puedan haber afectado los resultados del experimento son: los problemas de cobertura de Internet que dificultaban el acceso fluido de las estudiantes para visualizar los recursos virtuales; la falta de hábitos de estudio; el tiempo de exposición a los recursos; así como la autonomía para el aprendizaje, que se buscó reducir con la presencia de monitoras que hacían el seguimiento respectivo a los estudiantes para el ingreso al aula virtual. Estas consideraciones podrían explicar la situación de que en el postest no se superó la media del puntaje total de las pruebas aplicadas, a pesar de que en el postest los promedios están mucho mejor que en el pretest.

En ese sentido, se obtuvieron los siguientes resultados respecto de la parte descriptiva, el grupo experimental que fue expuesto al OVA “A” de interactividad pasiva, obtuvo en el pretest un puntaje promedio de 3,71 de un total de 18 puntos, a diferencia de los valores obtenidos en el post test, donde se evidencia un puntaje promedio de 8,68 puntos. Con relación a las dimensiones antes, durante y después de la lectura, en los pretest se obtuvieron promedios de $1,80,0,44$ y 1,46 respectivamente, mientras que en el postest los puntajes obtenidos fueron $3,80,1,71$ y 3,17 , evidenciándose una nítida mejora a través del uso de los diversos tipos de estrategias de lectura.

En cuanto al grupo experimental que usó el OVA "B" de interactividad limitada, el promedio obtenido de la variable dependiente "uso de estrategias de lectura" en el pre test es 3,83 de un total de 18 puntos, mientras que en el postest se obtuvo una media de 9,41. Sobre las tres dimensiones de la variable dependiente, estrategias antes de la lectura, durante la lectura y después de la lectura, el promedio de los datos obtenidos mejoró de 1,73 a 4,31 puntos, de 0,44 puntos a 0,57 puntos y de 1,56 a 3,24 puntos respectivamente.

Como ya se comentó, inclusive luego de la intervención, los puntajes obtenidos no superan la media en la mayoría de casos, del total del puntaje de la prueba para la variable y sus respectivas dimensiones, lo que se puede explicar por las condiciones iniciales en que se encontraba el grupo de estudiantes - bajos resultados en cuanto a comprensión lectora y en el 
Eficacia de los objetos virtuales para el aprendizaje en el uso de estrategias de lectura de estudiantes de distritos del Perú...

uso de estrategias para la comprensión-; probablemente con una intervención más extensa, los resultados podrían haber sido mejores. El estudio de Lozano (2013) con estudiantes del primer año de medicina en Ecuador tenía el propósito de identificar las estrategias metodológicas que poseen los estudiantes con el fin de comprender lo que leen, y halló que los estudiantes evidencian diversas carencias en estrategias para la comprensión y fluidez lectora y que, al menos el 75\% de ellos, tenían problemas de comprensión lectora, cuando se supone que por las exigencias de la formación en esa carrera profesional son estudiantes que ingresa con los mejores promedios y mayor amplitud de conocimientos a la universidad. De esa manera, se evidenció que a pesar de que los estudiantes en los países andinos están estudiando alguna carrera a nivel superior o en su defecto están culminando la educación básica regular, no poseen competencias básicas como el uso de herramientas básicas que les permitan desarrollar otras tareas más complejas como, en este caso, la comprensión de textos.

Al comparar los resultados obtenidos en el postest en los experimentos con los OVA A y B, y partiendo de que las condiciones iniciales de los grupos eran similares en cuanto al uso de las estrategias de lectura, se obtuvieron promedios de 8,68 y 9,41 de un total de 18 puntos. Con relación al comportamiento de las dimensiones de la variable: en estrategias antes de la lectura, el grupo experimental OVA A obtuvo 3,80 puntos promedio mientras que el grupo experimental OVA B 4,31. En las estrategias durante la lectura el GE OVA A, obtuvo 1,71 mientras que el GE con el OVA B, obtuvo 1,86 puntos. Finalmente, para la dimensión estrategias después de la lectura el GE OVA A, obtuvo 3,17 puntos frente al GE OVA B que obtuvo 3,24 puntos. Al realizar las comparaciones intergrupos, se halló que no existían diferencias significativas entre los dos grupos experimentales; es decir, el nivel de interactividad no significó mayores diferencias en el uso de estrategias de lectura. No obstante, en el análisis descriptivo, se pudo observar que las puntuaciones promedio del uso de estrategias de lectura del OVA B son mejores que los del OVA A.

Los hallazgos obtenidos coinciden con los trabajos de White y Sykes (2012); Snowball (2014); Feria y Zúñiga (2016); Castaño et al., (2014), quienes refieren que la interactividad de los contenidos es un aspecto importante para los resultados de aprendizaje, mientras que con respecto a la no existencia de diferencias significativas en los resultados, de acuerdo al tipo de interactividad aplicada en los recursos, resultados similares obtuvieron Guthrie et al. (2015), quienes hallaron efectos positivos en la resolución de problemas aplicando experimentos donde usaban tanto alta como baja interactividad, sin evidenciar diferencias significativas entre ellos.

Al observar su eficacia, se requiere mayores trabajos de investigación sobre diversos aspectos del diseño de recursos virtuales para el aprendizaje, especialmente en zonas donde los problemas de conectividad representan un inconveniente para realizar actividades con mayor interactividad. 


\section{Conclusiones}

Se demostró que, a través de la aplicación de objetos virtuales de aprendizaje, OVA, de interactividad pasiva así como de interactividad limitada, se logró mejoras estadísticamente significativas en el uso de las estrategias de comprensión lectora y, específicamente, en cada uno de los tipos de estrategias usadas antes, durante y después de la lectura.

No se evidenciaron diferencias significativas en los resultados obtenidos, luego de aplicar los objetos virtuales para el aprendizaje de ambos tipos en las respectivas muestras y evaluar la variable dependiente uso de estrategias de lectura, así como cada una de sus dimensiones, concluyéndose que aspectos como la interactividad en los OVA utilizados en este estudio, no han significado mayores diferencias en la adquisición y puesta en práctica de las estrategias de lectura. Sin embargo, en los resultados descriptivos se evidenciaron que a través de los OVA de interactividad limitada se obtuvo mejores resultados mediante la interactividad pasiva.

\section{Referencias}

Banco Mundial. (2018). Global knowledge index-scorecard. Recuperado de http: / / knowledge4all. $\mathrm{com} /$ ScoreCard2018. aspx id $=1086$ \&language $=\mathrm{en}$.

Castaño, J., Duart, J. y Sancho, T. (2015). Determinantes del uso de Internet para el aprendizaje interactivo: un estudio exploratorio. New Approaches in Educational Research, 4(1), 25-34.

Castillo, É. Y., García, N. N., Corredor, C. M., \& Malaver, C. E. C. (2017). Evolución de la educación superior a distancia: desafíos y oportunidades para su gestión. Revista Virtual Universidad Católica del Norte, 50, 80-105. Recuperado de http://revistavirtual.ucn.edu. co/index.php/RevistaUCN/article/view/814/1332

Feria, I. y Zúñiga, K. (2016). Objetos virtuales de aprendizaje y el desarrollo del aprendizaje autónomo en el área de inglés. Praxis, 12, 63-77.

Guthrie, L., Vallée-Tourangeau, F., Vallée-Tourangeau, G. \& Howard, C. (2015). Learning and interactivity in solving a transformation problem. Memory \& Cognition, 43(5). 723-735. Recuperado de https: / link.springer.com/article/10.3758/s13421-015-0504-8.

Gutiérrez, C. y Salmerón, H. (2012). Estrategias de comprensión lectora: enseñanza y evaluación en educación primaria. Revista de Currículum y Formación del Profesorado, 16(1), 183-202.

IMD World Competitiveness Center. (2018). IMD World Digital Competitiviness Ranking 2018. Recuperado de https://bit.ly/2t4oFcb. 
Eficacia de los objetos virtuales para el aprendizaje en el uso de estrategias de lectura de estudiantes de distritos del Perú...

Lozano, C. M. (2013). Estrategias metodológicas de la lectura y la potenciación de la comprensión lectora. Propuesta: guía didáctica para primer año de medicina de la Universidad de Guayaquil (tesis de maestría). Universidad de Guayaquil, Guayaquil, Ecuador.

Minedu. (2017). Resultados de la evaluación censal ECE 2016. Recuperado de http://umc. minedu.gob.pe/resultadosece2016/.

Ruiz, C. (1992). Instrumentos y técnicas de investigación educativa: un enfoque cuantitativo y cualitativo para la recolección y análisis de datos. Recuperado de https: / / goo.gl/Tg1f23.

Sánchez, A. (2009). Taller de lectura y redacción 1. Ciudad de México, México: Cengage Learning.

Snowball, S. D. (2014). Using interactive content and online activities to accommodate diversity in a first year class. Springer Science Business Media Dordrecht. Recuperado de: https: / /link.springer.com/article/10.1007/s10734-013-9708-7.

Solé, I. (2011). Estrategias de lectura. Barcelona, España: Editorial Graó.

White, S. \& Sykes, A. (2012). Evaluation of a blended learning approach used in an anatomy and physiology module for pre-registration healthcare students. International Conference on Mobile, Hybrid, and On-line Learning, 1-9. 